\title{
Image Recapture Discovery Algorithms: A Survey
}

\author{
Pradnya Sanas ${ }^{1}$, Poonam Gupta ${ }^{2}$ \\ ${ }^{1}$ Pune University, G.H.R.C.E.M, Wagholi, Pune, Maharashtra, India
}

${ }^{2}$ Assistant Professor, Pune University, G.H.R.C.E.M, Wagholi, Pune, Maharashtra, India

\begin{abstract}
With the development of multimedia technology and digital devices, it is very easier to recapture image of high quality from LCD screens and the use of such recapture image in authentication can be harmful. An attacker may choose to recapture a forged image in order to hide imperfections and to increase its authenticity. To do forensic analysis of such images for evidence is very important. So detection of recapture images from set of images is very important in this case. In this paper we did survey of various recapture image detection algorithms with their pros and cons. Also we studied various features like aliasing, blurriness etc. of recaptured images, which can differentiate recapture image from original one.
\end{abstract}

Keywords: Multimedia Technology, LCD, Image Forensic, Recapture Detection, Aliasing, Blurriness.

\section{Introduction}

To restore the trustworthiness of digital images, image forgery detection [10] has been intensively studied in recent years through detection of certain intrinsic image regularities or some common tampering anomalies. Frequently, the telltale cues useful for image forensics such as lens distortion, sensor noise pattern and statistics, demos icing regularity and JPEG characteristics are directly associated with the image creation pipeline, where the light signals are converted into a digital image. Though some forensic methods can efficiently expose the direct tampering made on an image, most existing methods are unable to expose the indirect scenery forgery, where the scenery to be captured is artificially created. Though creating physical scenery in general can be a very difficult and expensive task, with the aid of today's ubiquitous and high-fidelity display technology, generating virtual scenery of reasonable fidelity is still relatively easy and such technology is potentially exploited to defeat the current image forensics systems.

Traditionally, photographs have been associated with a high degree of authenticity and were considered difficult to forge. With the advent of digital photography image tampering is now commonplace and can easily be performed using commercial, widely available, image editing software [3]. In practice, unless an attacker is highly skilled, imperfections in the forged image may be present and the attacker may attempt to conceal them by recapturing the forged image from an LCD monitor. By recapturing the image, an additional level of authenticity, typically associated with a single captured image, is introduced into the forgery making it more difficult to detect. For this reason this paper review various solutions to problem of detecting whether a given image was recaptured with a digital still camera from an image displayed on an LCD monitor or whether it was a single capture of a natural scene.

\section{Features of Recaptured Images}

In this section we provide an overview of some of the more common features found in images that have been recaptured from LCD monitors.

\subsection{Aliasing}

Aliasing is sometimes introduced in digital camera images when the scene is insufficiently band-limited or contains detail with very high spatial frequencies [20]. In cameras that are equipped with a Color Filter Array (CFA) [21] the color channels are normally sampled at frequencies that are lower than the native frequency of the image sensor.

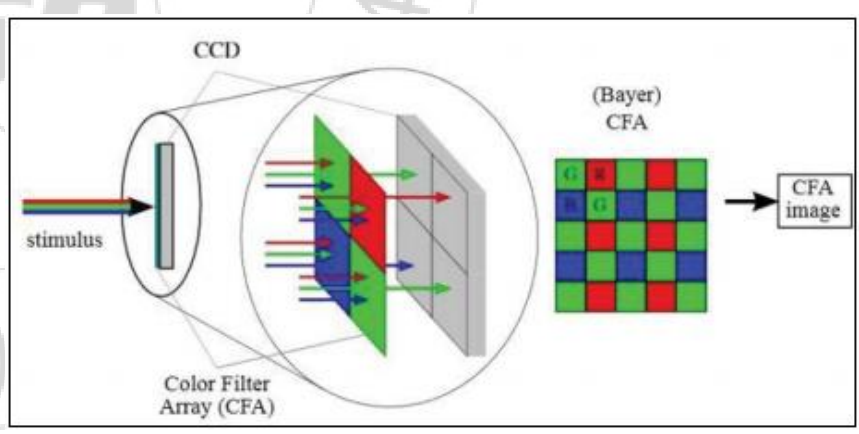

Figure 1: Bayes Color Filter Array

The recapture of an image displayed on the screen of an LCD monitor is, therefore, highly likely to introduce aliasing due to the high frequency periodic pattern of the monitor pixel grid structure. Indeed, casually recaptured still images or videos of LCDs are often characterized by the presence of aliasing artifacts, also referred to as color moiré, over the visible region of the display.

\subsection{Blurriness}

There are three possible scenarios that blurriness can arise in a recaptured image. First, the first capture device or the printing device could be of low resolution. Second, the attack image may be small and the display medium may have to be placed outside of the focus range due to a specific recaptured 


\section{International Journal of Science and Research (IJSR) \\ ISSN (Online): 2319-7064}

Index Copernicus Value (2013): 6.14 | Impact Factor (2014): 5.611

setting. One way of characterizing the blur is with the point spread function (PSF) of the capture device. In practice measuring the PSF of a device is not easily achieved and the line spread function (LSF) is used instead.

\subsection{Contrast, Color and Illumination Non-Uniformity}

Because the light transmitted from the back can significantly reduce the contrast and saturation of a recaptured image, the color of finely recaptured images still looks different from their original images. Contrast and color moments for an image can be computed as a distinguishing feature. Color balance errors in a recaptured image can be minimized by calibrating the display monitor and by presetting the white point of the recapture camera to the LCD monitor white point before recapture.

A luminance gradient may be noticeable in recaptured images containing large regions that are low in texture or detail. Identification of the luminance gradient would enable recaptured images to be detected.

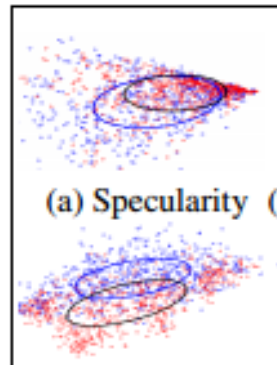

(d) Contrast

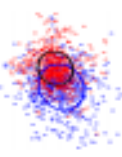

(b) Surface Gradient

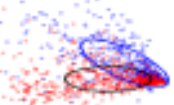

(e) Chromaticity

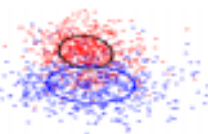

(c) Color Histogram

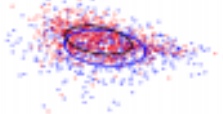

(f) Blurriness
Figure 2: 2D projection of the physical feature distribution for the real-scene image sets (red) and the recaptured image sets (blue).

\section{Existing System}

\subsection{An Investigation into Aliasing In Images Recaptured From An LCD Monitor Using A Digital Camera [2]:}

Hani Muammar and Pier Luigi Dragotti investigated one approach to detecting an image that has been recaptured from an LCD monitor is to search for the presence of aliasing due to the sampling of the monitor pixel grid. An analysis of aliasing in recaptured images of LCD monitors using digital cameras equipped with a Bayer CFA was presented. The periodic structure of the monitor pixel grid projected on the camera's image sensor was modelled in one dimension by a 2-dimensional square wave.

In this paper they show that aliasing can be completely eliminated in a recaptured image by setting the camera to monitor distance to a value determined by the camera lens focal length, the pixel pitch of the LCD monitor and the pixel pitch of the camera's image sensor. A recapture detector should not therefore rely solely on the presence of aliasing, but should make use of other features present in recaptured images such as high scene tonal contrast, changes in color balance and loss in perceived sharpness.
Advantages:

- Very effective technique to eliminate aliasing effect from recaptured images.

Disadvantages:

- Paper investigation is in aliasing only.

- Camera dependency.

- User should have knowledge about technical details of captures of camera.

\subsection{An Image Recapture Detection Algorithm Based on} Learning Dictionaries of Edge Profiles [1]:

Thirapiroon Thongkamwitoon, Hani Muammar, and PierLuigi Dragotti proposed algorithm to detect recapture image based on learning dictionaries of edge profiles. They proposed a method for image recapture detection based on the blurriness of edges.
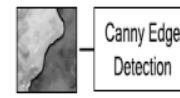
Detection Input Image

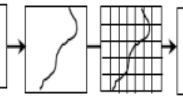
Detection

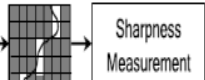
Extracted Line Spread Binary Block-based Mask computation Neasurement Blocks Profile Matrix

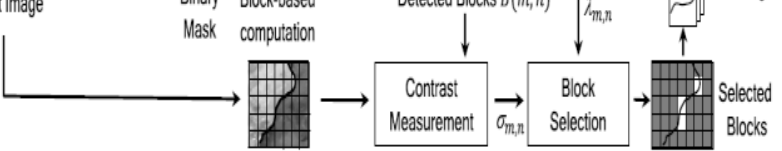

Figure 3: Working diagram of the automatic block-based edge detection algorithm.

\section{Algorithm 1:}

Step 1: Firstly, the query image is converted to greyscale.

Step 2: all edges contained in the image are detected using a Canny Edge Detector.

Step 3: Edge profiles are extracted locally

The query image is divided into a number of non-overlapping square blocks $\mathrm{B}(\mathrm{m}, \mathrm{n})$ of size $\mathrm{W} \times \mathrm{W}$ with $\mathrm{W}=16$ pixels. Here $m$ and $n$ are the vertical and horizontal indices of the block respectively.

Step 4: For each block, first check whether it contains a horizontal or near horizontal sharp single edge.

Step 5: The block will be detected only when the condition $\eta$ $\geq \beta W, \beta=0.6$

Step 6: The detected blocks, B $(m, n)$, are then ranked according to their sharpness and edge contrast.

Block sharpness is determined using the technique in which the average width ${ }^{-} \lambda \mathrm{m}, \mathrm{n}$ of line spread profiles of the Blocks are estimated.

The contrast of a block is measured by computing the blockbased variance, $\sigma \mathrm{m}, \mathrm{n}$, of the input image at the detected block.333

Step 7: Create Feature matrix:

let $\mathrm{Y} \in \mathrm{IRW} \times \mathrm{W}$ be a matrix which represents the grey scale values of a block. Each column, yi ; i = 1, 2, $\cdots, \mathrm{W}$, of the matrix Y may, therefore, be considered to represent an edge profile of the image.

This feature matrix is used for training and testing purposes. Here authors used SVM classification algorithm for labeling to images. 


\section{International Journal of Science and Research (IJSR) \\ ISSN (Online): 2319-7064}

Index Copernicus Value (2013): 6.14 | Impact Factor (2014): 5.611

Advantages:

- Very good success rate for dataset.

Disadvantages:

- Need to remove aliasing factor from image.

- Technique work for images only

\subsection{Robust Image Recapture Detection Using K-SVD Learning Approach To Train Dictionaries Of Edge Profiles}

Thirapiroon Thongkamwitoon, Hani Muammar, and Pier Luigi Dragotti show that it is possible to detect a recaptured image from the unique nature of the edge profiles present in the image. They leverage the fact that the edge profiles of single and recaptured images are markedly different and they train two alternative dictionaries using the KSVD approach. One dictionary is trained to provide a sparse representation of single captured edges and a second for recaptured edges. Using these two learned dictionaries, they can determine whether a query image has been recaptured. They achieve this by observing the type of dictionary that gives the smallest error in a sparse representation of the edges of the query image.

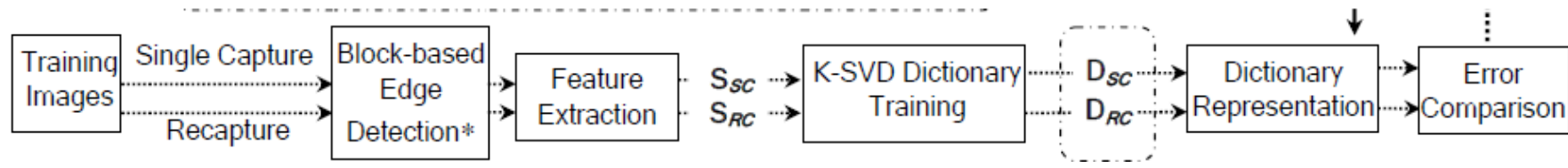

Figure 4: Flow of Recapture detection

\section{Algorithm 2:}

Step 1: We trained two dictionaries DSC and DRC using features SSC and SRC extracted from the single captured and recaptured images respectively.

Step 2: Each dictionary is considered to provide an optimal representation of the profiles extracted from edges found in each set of training images, respectively.

Step 3: We now assume that a query image containing edges is available (Algorithm 1).

Step 4: Given the matrix $\mathrm{Q} \in \mathrm{IRW}-1 \times \mathrm{N}$ which represents all line spread profiles extracted from the detected blocks, the decision for recapture detection can be based on the class of dictionary that gives the smallest representation error.

Step 5: We defineX1 andX2 as the coefficient matrices obtained from the composition of query feature matrix $Q$ using the dictionaries DRC and DSC respectively.
Step 6: The query image is classified into a recapture group if Otherwise, the query image is classified to the single capture group.

$$
\left\|\mathbf{Q}-\mathbf{D}_{R C} \mathbf{X}_{1}\right\|_{F}^{2}<\left\|\mathbf{Q}-\mathbf{D}_{S C} \mathbf{X}_{2}\right\|_{F}^{2}
$$

Advantages:

- Method does not require human supervision.

- The does not need presence of sharp edges in query images.

Disadvantages:

- Need to train dictionaries.

\section{System Architecture}

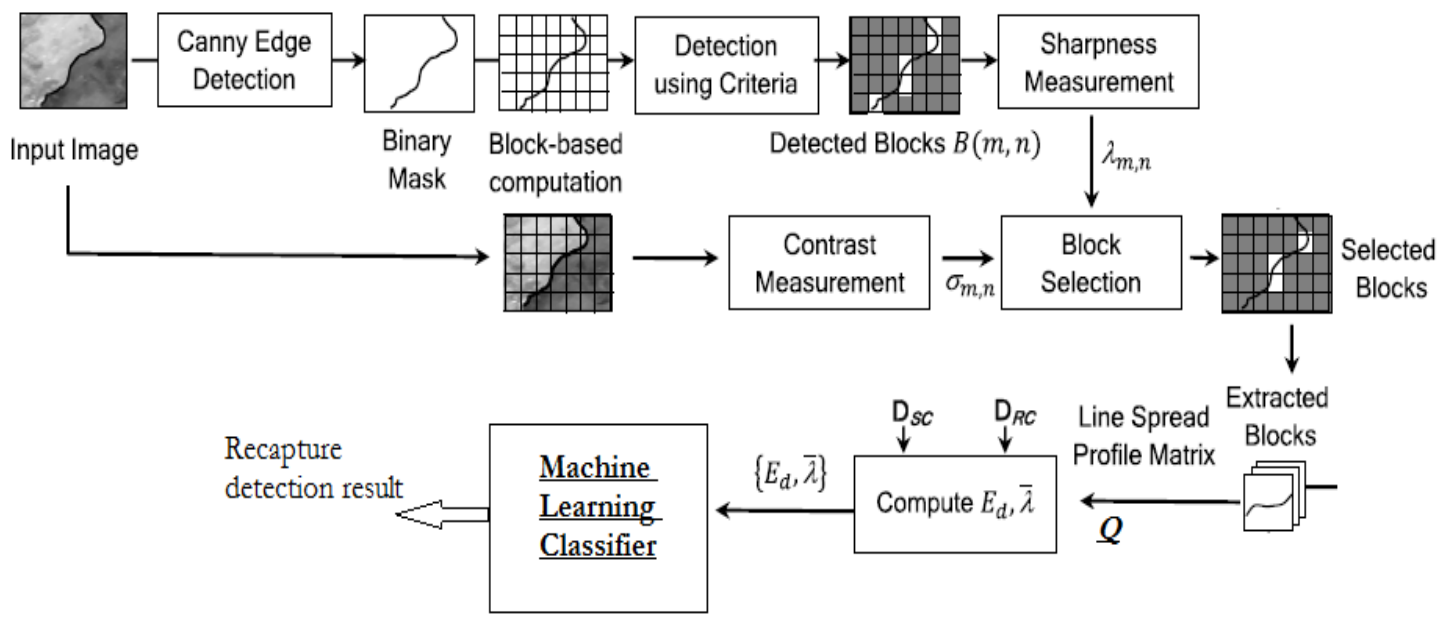

Figure 5: System Architectureof the image classification techniques

A method that uses the edge blurriness and distortion introduced by the recapture process as a feature to detect if a given image has been recaptured from an LCD monitor. We show that the edges found in single and recaptured images 


\section{International Journal of Science and Research (IJSR) \\ ISSN (Online): 2319-7064}

Index Copernicus Value (2013): 6.14 | Impact Factor (2014): 5.611

can be fully characterized by their line spread function (LSF). We then describe how sets of elementary atoms that provide a sparse representation of LSFs can be learned using the KSVD dictionary learning method [19]. Specifically, a singlecapture dictionary is created from a training set of single captured images and a second one from recaptured images. We also compute an edge spread width from the line spread function of the image and combine this feature with the dictionary approximation errors to train an SVM classifier. We classify a query image as single or recaptured depending on its location relative to the SVM hyper plane.

\section{Conclusion}

In this paper, we study various features which can differentiate original images from recaptured one like aliasing, blurriness, noise, surface gradient etc. Using these features many researchers proposed new algorithms to detect recaptured images or videos. So here we study algorithms of many researchers who used different features for detection of recapture image.

As future work, it will be interesting to analyze more feature of image which can be useful for classification. Also no author works on 3D- images, so this is another interesting direction to proceed this work on $3 \mathrm{D}$ images. As per previous work for classification of images many authors used SVM algorithm, so it will another direction for researcher to do more analysis on another machine learning algorithms for better result.

\section{Acknowledgment}

We would like to thank all the authors of different research papers referred during writing this paper. It was very knowledge gaining and helpful for the further research to be done in future.

\section{References}

[1] Thirapiroon Thongkamwitoon, Hani Muammar, PierLuigi Dragotti, "An Image Recapture Detection Algorithm Based on Learning Dictionaries of Edge", in IEEE Transaction On Information Forensic And Security, VOL. 10, NO. 5, MAY 2015

[2] H. Muammar and P. L. Dragotti, "An investigation into aliasing in images recaptured from an LCD monitor using a digital camera," in Proc. IEEE Int. Conf. Acoust., Speech Signal Process. (ICASSP), Vancouver, BC, Canada, May 2013, pp. 2242-2246.

[3] J. Yin and Y. Fang, "Markov-based image forensics for photographic copying from printed picture," in Proc. 20th ACM Int. Conf. Multimedia (MM), Nara, Japan, 2012, pp. 1113-1116.

[4] J. Bai, T.-T. Ng, X. Gao, and Y.-Q. Shi, "Is physicsbased liveness detection truly possible with a single image?" in Proc. IEEE Int. Symp. Circuits Syst. (ISCAS), Jun. 2010, pp. 3425-3428.

[5] X. Gao, T.-T. Ng, B. Qiu, and S.-F. Chang, "Single-view recaptured image detection based on physics-based features," in Proc. IEEE Int.Conf. Multimedia Expo (ICME), Jul. 2010, pp. 1469-1474.

[6] H. Cao and A. C. Kot, "Identification of recaptured photographs on LCD screens," in Proc. IEEE Int. Conf. Acoust. Speech Signal Process. (ICASSP), Mar. 2010, pp. 1790-1793.

[7] J. Yin and Y. Fang, "Digital image forensics for photographic copying," Proc. SPIE, vol. 8303, pp. 1-7, Feb. 2012, Art. ID 83030F.

[8] Y. Ke, Q. Shan, F. Qin, and W. Min, "Image recapture detection using multiple features," Int. J. Multimedia Ubiquitous Eng., vol. 8, no. 5, pp. 71-82, 2013.

[9] X. Gao, B. Qiu, J. Shen, T.-T. Ng, and Y. Q. Shi, “A smart phone image database for single image recapture detection," in Proc. 9th Int.Workshop Digit. Watermarking (IWDW) Revised Select. Papers, 2011, pp. 90-104.

[10] N. Kose and J. Dugelay, "Classification of captured and recaptured images to detect photograph spoofing," in Proc. Int. Conf. Informat.,Electron. Vis. (ICIEV), May 2012, pp. 1027-1032.

[11]A. da Silva Pinto, H. Pedrini, W. Schwartz, and A. Rocha, "Video-based face spoofing detection through visual rhythm analysis," in Proc. 25th SIBGRAPI Conf. Graph., Patterns Images (SIBGRAPI), Aug. 2012, pp. 221-228.

[12]J.-W. Lee, M.-J. Lee, T.-W. Oh, S.-J. Ryu, and H.-K. Lee, "Screenshot identification using combing artifact from interlaced video," in Proc. 12th ACM Workshop Multimedia Secur. (MM\&Sec), Rome, Italy, 2010, pp. 49-54.

[13]P.Bestagini, M. Visentini-Scarzanella, M. Tagliasacchi, P. L. Dragotti, and S. Tubaro, "Video recapture detection based on ghosting artifact analysis," in Proc. 20th IEEE Int. Conf. Image Process. (ICIP), Sep. 2013, pp. 4457-4461.

[14] M. Visentini-Scarzanella and P. L. Dragotti, "Video jitter analysis for automatic bootleg detection," in Proc. IEEE 14th Int. Workshop Multimedia Signal Process. (MMSP), Sep. 2012, pp. 101-106. I. 